\title{
Circulating levels of biologically active parathyroid hormone in rheumatic diseases
}

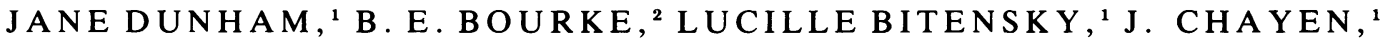 \\ N. LOVERIDGE, ${ }^{1}$ AND J. T. SCOTT ${ }^{2}$ \\ From the ${ }^{1}$ Division of Cellular Biology, Kennedy Institute of Rheumatology, Bute Gardens, London W6 7DW, \\ and the ${ }^{2}$ Kennedy Institute of Rheumatology and Charing Cross Hospital, London W6
}

SUMmARY There has been doubt as to whether elevated levels of parathyroid hormone, reported previously by radioimmunoassay, reflect increased concentrations of the biologically active hormone. The application of a recently developed, highly sensitive bioassay has shown considerable disparity between bioactivity and immunoreactivity in 5 rheumatic conditions and in normal subjects. Six patients with chondrocalcinosis had elevated levels; 3 of these did not have hypercalcaemia or any obvious cause other than possible subclinical hyperparathyroidism. One patient, assayed during an acute episode, had an elevated concentration of the hormone which reverted to normal when she was asymptomatic. Most patients with osteoarthrosis (13 out of 15) had low normal levels; 2 showed unexplained slightly elevated concentrations. Of 6 patients with haemochromatosis 3 had elevated levels, though this may have been related to the associated presence of diabetes mellitus. A third of patients with ankylosing spondylitis (10 out of 30) showed elevated parathyroid hormone levels but without hypercalcaemia. A number of spondylitic patients also showed anomalous results in this assay, possibly due to the presence of an antagonist. This would be consistent with the absence of clinical or biochemical evidence of hyperparathyroidism.

An association between hyperparathyroidism and calcium pyrophosphate deposition in articular cartilage (and pseudogout) is now well recognised, ${ }^{1-4}$ although hyperparathyroidism is generally considered to be a rare cause of chondrocalcinosis. However, Phelps and Hawker, ${ }^{5}$ studying immunoreactive parathyroid hormone (PTH) levels in 26 patients with calcium pyrophosphate dihydrate deposition disease (CPPD), found elevated levels in 10, 4 of whom had clinical and biochemical evidence of primary hyperparathyroidism and 3 had presumed secondary hyperparathyroidism. These findings were supported by McCarty et al., ${ }^{2}$ who, again using a radioimmunoassay, found elevated levels of PTH in 15 of 22 patients with chondrocalcinosis, but they also found elevated levels in over half their control group of patients with osteoarthrosis. Fairney et al.$^{6}$ studied immunoreactive PTH levels in 50 patients with ankylosing spondylitis and found that the mean level was elevated compared with a group of normal controls. Although there was no difference in the mean level of serum calcium between these 2 groups, the mean alkaline phosphatase was significantly higher in the

Accepted for publication 23 October 1981.

Correspondence to Dr J. Chayen. spondylitic group. They suggested that since there was no other clinical or biochemical evidence of hyperparathyroidism these results indicated a lack of end-organ responsiveness to PTH.

Intact parathyroid hormone, a polypeptide of 84 amino acids, has a half-life in the circulation of less than 10 minutes. The 1-34 amino acid sequence of the molecule is responsible for all the biological activity and also has a short circulating half-life, whereas the 35-84 sequence, either in its intact form or in fragments, is more antigenic and may accumulate in the circulation until cleared, principally by the kidneys. It is towards these more antigenic parts of the molecule that most antibodies used in immunoassays have been directed, and levels as high as $200-500$ $\mathrm{pg} / \mathrm{ml}$ have been found in normal individuals; indeed, Fairney et al. ${ }^{6}$ found the mean level for their normal group to be $746 \mathrm{pg} / \mathrm{ml}$.

Because these radioimmunoassays mainly measured the split products of the hormone, McCarty et al. ${ }^{2}$ concluded that 'There is no way at present to determine whether the increased activity' (which they reported) 'represents the biologically active fragment'. The calculations of Parsons et al. ${ }^{7}$ that the circulating level of the biologically active 
hormone cannot exceed about $20 \mathrm{pg} / \mathrm{ml}$ reinforces this doubt as to the meaning of the radioimmunoassays. The development of a highly sensitive cytochemical bioassay, ${ }^{8}$ which has been substantiated in other laboratories, ${ }^{910}$ made it feasible to investigate whether or not PTH levels varied in these rheumatological disorders.

\section{Patients and methods}

Mid-morning samples, obtained from all subjects without previous fasting, were taken into lithium heparin tubes; the plasma was separated immediately, snap-frozen to $-70^{\circ} \mathrm{C}$, and stored at this temperature. All samples were assayed by the cytochemical bioassay within 2 weeks against the bovine 1-84 parathyroid hormone reference standard (NIBSC code 77/533). One hundred and eight samples were assayed from a selection of patients with well characterised rheumatic conditions and from normal volunteers.

Plasma creatinine (normal range $42-130 \mu \mathrm{mol} / \mathrm{l}$ ), serum calcium (normal range $2 \cdot 2-2 \cdot 6 \mathrm{mmol} / \mathrm{l}$ ), and alkaline phosphatase (normal range 3-13 KA units) were assayed by standard AutoAnalyzer techniques in the routine Chemical Pathology Laboratory at the Charing Cross Hospital.

Chrondrocalcinosis. Twenty-two samples were obtained from 21 patients with chondrocalcinosis $(15$ females, 6 males; mean age: 73 years; range: $48-94$ ). All had a history or clinical evidence of synovitis of at least one joint. Four patients had a history of acute monoarticular pseudogout, and in 3 of these calcium pyrophosphate dihydrate crystals were identified by polarised light microscopy in synovial fluid; in 2 patients crystals were also found in synovial biopsies taken at arthroscopy. Two patients had a history of polyarthritis, and one was assayed during and after an acute exacerbation. All patients had radiological chondrocalcinosis of at least 2 joints, and the mean number of radiologically involved joints for the group was 6 joints per patient. Patients were excluded from this group if they had obvious evidence of generalised osteoarthrosis. So far as possible patients with other diseases, particularly renal failure, were excluded, with the exception of one patient with acute pseudogout and widespread chondrocalcinosis who had advanced renal failure and another with a slightly elevated plasma creatinine level. One further patient in this group was subsequently found to have a pancreatic tumour with metastases, but his chondrocalcinosis had predated this by some 6 years. Two patients had hypercalcaemia, but neither had clinical or radiological features of hyperparathyroidism, and PTH levels measured previously by immunoassay had been normal. None of these patients had any clinical or laboratory evidence of haemochromatosis or diabetes mellitus.

Haemochromatosis. Six male patients with haemochromatosis were studied (age range 45-73 years; mean 65 years); 4 had chondrocalcinosis and 3 of these and one other had insulin-dependent diabetes mellitus.

Osteoarthrosis. Fifteen patients with uncomplicated generalised osteoarthrosis were studied (11 females, 4 males), all with normal renal function and serum calcium and no evidence of radiological chondrocalcinosis. In each patient a minimum of 5 small or 3 large joints were involved both clinically and radiologically; the mean number of joints involved was 14 joints per patient. The patients' ages ranged from 53 to 80 years (mean age 65 years).

Ankylosing spondylitis. Thirty patients fulfilling the New York criteria ${ }^{11}$ for definite ankylosing spondylitis were included. There were 24 men and 6 women, their ages ranging from 24 to 86 years (mean age 46 years). Twenty-seven possessed the histocompatability antigen HLA B27, and the duration of the disease varied from 6 months to 32 years (mean $13 \cdot 6$ years).

Sixteen patients had peripheral arthritis and 9 had extra-articular features of the disease. None had radiological chondrocalcinosis. Disease activity was graded $0-4$ on a clinical scoring system (scoring one each for back pain or morning stiffness, peripheral arthritis, extra-articular features, ESR $>25 \mathrm{~mm} / \mathrm{h}$, or anaemia). All these patients had normal levels of plasma creatinine, serum calcium, and alkaline phosphatase.

Rheumatoid arthritis. Eight patients fulfilling the American Rheumatism Association criteria ${ }^{12}$ for classical or definite rheumatoid arthritis were included ( 6 females, 2 male). Their ages ranged from 53 to 74 years (mean 58 years). All these patients had normal levels of plasma creatinine, serum calcium, and alkaline phosphatase.

Healthy persons. Twenty-seven healthy adult volunteers were also studied (12 male, 15 female). Their ages ranged from 21 to 79 years (mean 44 years).

\section{Results}

The levels of circulating bioactive parathyroid hormone are shown in Fig. 1. The normal range for this assay is $0 \cdot 2-12.0 \mathrm{pg} / \mathrm{ml}(=\mathrm{ng} / \mathrm{l})$ with a mean of $2 \cdot 9 \pm 2 \cdot 83 \mathrm{pg} / \mathrm{ml}(\mathrm{ng} / \mathrm{l})$; this approximates to the previously expected range of bioactivity found in the experiments of Parsons et al. ${ }^{7}$ and to the normal range found by other workers. ${ }^{910}$ All the samples with the exception of a few from patients with ankylosing spondylitis showed a good parallelism of response at 


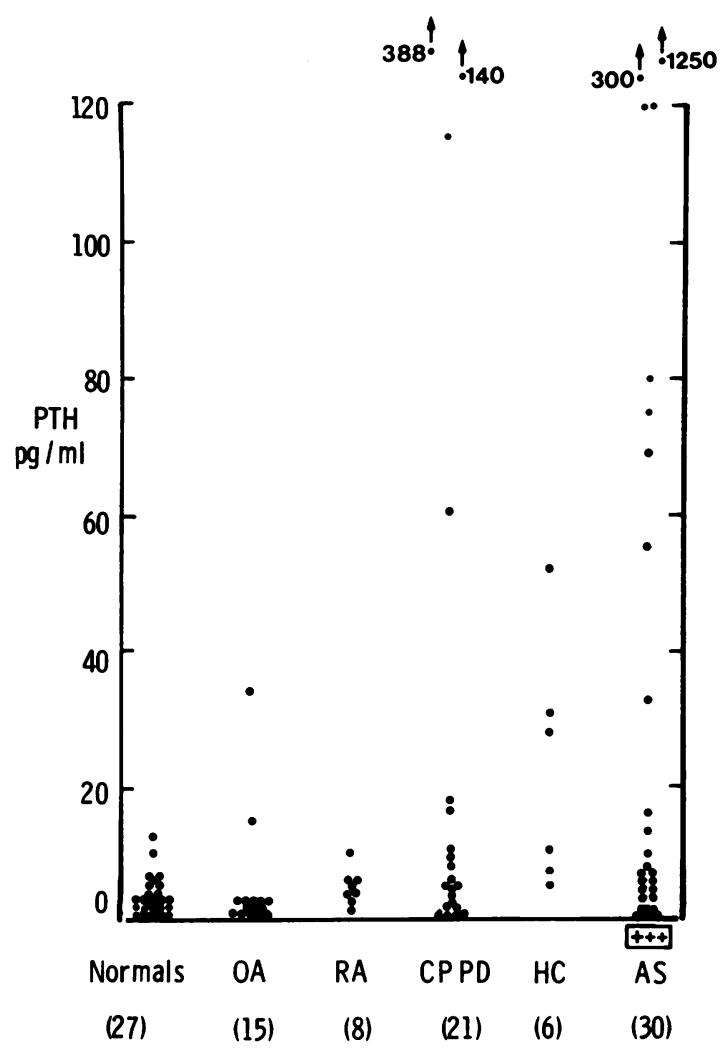

Fig. 1 Circulating levels of bioactive parathyroid hormone in 27 normal subjects, 15 patients with osteoarthrosis $(O A)$, 8 with rheumatoid arthritis $(R A), 21$ with chondrocalcinosis (CPPD), 6 with haemochromatosis $(H C)$, and 30 with ankylosing spondylitis $(A S)$. + Indicates samples giving totally anomalous results.

routine plasma dilutions against the standard calibration graph.

Osteoarthrosis. Of the 15 patients with osteoarthrosis 13 had PTH values of less than $4 \mathrm{pg} / \mathrm{ml}$ (ng/l), but the remaining 2 patients had elevated levels, one of $15 \mathrm{pg} / \mathrm{ml}(\mathrm{ng} / \mathrm{l})$ and the other of 34 $\mathrm{pg} / \mathrm{ml}(\mathrm{ng} / \mathrm{l})$. Levels of alkaline phosphatase were normal apart from one patient with a value of $16 \mathrm{KA}$ units which was hepatic in origin and assumed to be due to antirheumatic therapy which the patient was receiving.

Chondrocalcinosis. Of the 22 samples from patients with chondrocalcinosis 16 had normal PTH levels and 6 were elevated, 4 of these above $60 \mathrm{pg} / \mathrm{ml}$ $(\mathrm{ng} / \mathrm{l})$, well into the range found in established primary hyperparathyroidism; ${ }^{8}$ the highest level, of 388 $\mathrm{pg} / \mathrm{ml}(\mathrm{ng} / \mathrm{l})$, was found in the patient with chronic renal failure and presumed secondary hyperparathyroidism. Another high level of $115 \mathrm{pg} / \mathrm{ml}$ (ng/l) was found in the patient with a pancreatic tumour. A third patient, with a level of $140 \mathrm{pg} / \mathrm{ml}$ (ng/l), had hypercalcaemia but without clinical or biochemical evidence of hyperparathyroidism and a normal level of PTH by radioimmunoassay on 3 occasions previously. However, apart from these patients there remained 3 others with elevated PTH values without clinical or other biochemical evidence of hyperparathyroidism.

In this group as a whole there was no correlation between PTH levels measured by bioassay and levels of plasma creatinine, serum calcium, or alkaline phosphatase. One patient in this group had 2 PTH estimations performed. This patient was first seen with an acute episode of polyarthritis; the PTH level was $60 \mathrm{pg} / \mathrm{ml}$ (ng/l). Eight months later, following treatment with anti-inflammatory drugs, she was virtually asymptomatic and the PTH level was then $2 \cdot 3$ $\mathrm{pg} / \mathrm{ml}(\mathrm{ng} / \mathrm{l})$.

Haemochromatosis. Of the 6 patients with haemochromatosis 3 had elevated PTH levels. All 3 had diabetes but only 2 of the 3 had chondrocalcinosis. The mean PTH level for the group was $22 \cdot 2 \mathrm{pg} / \mathrm{ml}(\mathrm{ng} / \mathrm{l})( \pm 17 \cdot 8)$, and this was significantly different from the control group $(p<0.001)$ and from the osteoarthrosis group $(\mathrm{p}<0.02)$ All 6 patients in

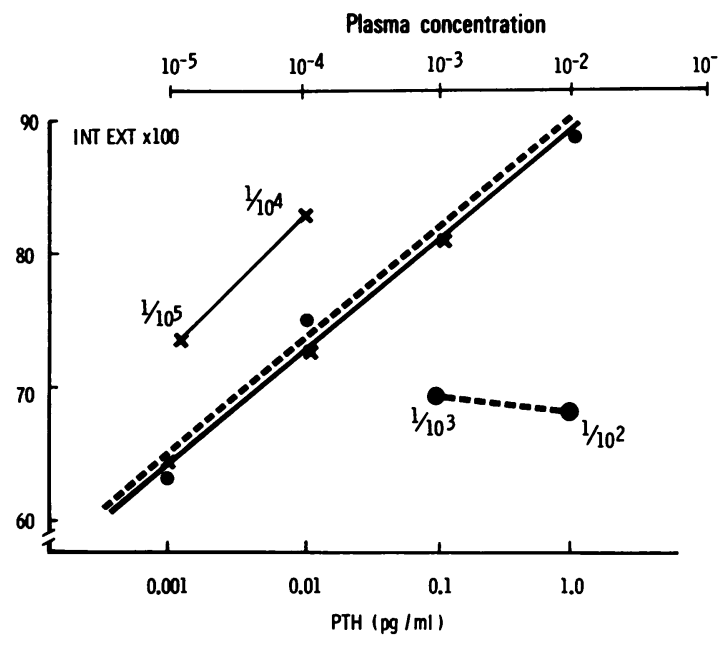

Fig. 2 Two assays performed on a plasma that initially produced an anomalous response. In the first assay (broken lines and solid circles) this plasma, at dilutions of $1 / 10^{2}$ and $1 / 10^{3}$ gave a flat response almost perpendicular to the calibration graph. However, in the second assay (solid line and crosses) with its own calibration graph (which was almost identical to that of the first assay), this plasma, now diluted $1 / 10^{4}$ and $1 / 10^{5}$, gave responses parallel to those of the calibration graph. 


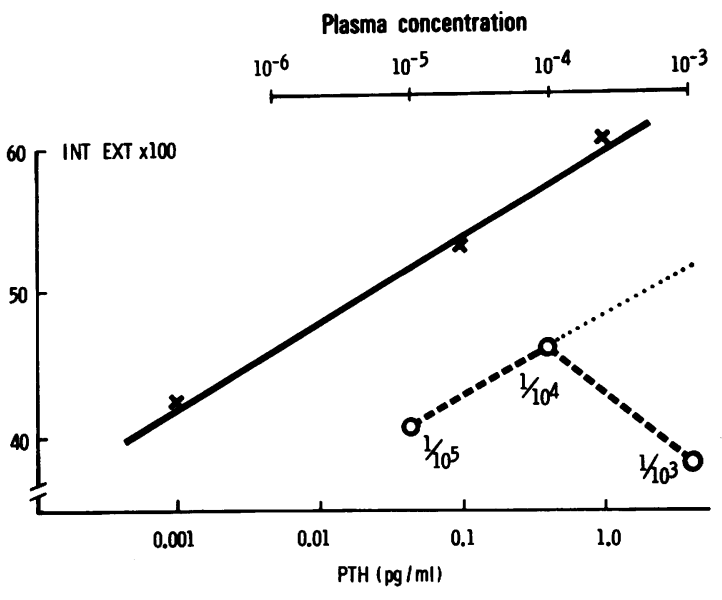

Fig. 3 The assay of one of the 3 apparently anomalous samples that assayed at high levels. In this plasma the $1 / 10^{3}$ and $1 / 10^{4}$ dilutions gave an anomalous result; at the higher dilutions $\left(1 / 10^{4}\right.$ and $\left.1 / 10^{5}\right)$ there was good parallelism (broken line and open circles). From these dilutions the circulating level of the hormone was $55 \mathrm{pg} / \mathrm{ml}(\mathrm{ng} / \mathrm{l})$. The anomalous result at a dilution of $1 / 10^{3}$ could not have been due to an excessive concentration of the hormone at this dilution. As shown by the dotted extrapolation from the line joining the results with $1 / 10^{5}$ and $1 / 10^{4}$ a 10 -fold increase in concentration would still have fitted within the calibration graph (solid line and crosses).

this group had normal levels of plasma creatinine, serum calcium, and alkaline phosphatase.

Rheumatoid arthritis. All 8 patients with rheumatoid arthritis had normal plasma PTH levels with a mean of $4.9 \pm 2.67 \mathrm{pg} / \mathrm{ml}$ (ng/l) (Fig. 1).

Ankylosing spondylitis. Of the 30 patients in this group $17(57 \%)$ had PTH levels within the normal range. Ten assayed high: in 6 of these the plasma had to be diluted to $1: 10^{4}$ and $1: 10^{5}$ to bring the concentration of the hormone to within the calibration range of the assay (Fig. 2) so as to produce a parallel response, as is required in bioassay. In 4 of these 10 , although a parallel response was achieved with increased dilution, the calculated level was insufficient to account for the loss of parallelism at the higher concentrations (Fig. 3). The other 3 samples gave totally anomalous and low assays, suggestive of some inhibitory influence.

Normal PTH levels were found in all the female patients, with one being marginally elevated at 13.5 $\mathrm{pg} / \mathrm{ml}(\mathrm{ng} / \mathrm{l})$. Of the 3 patients who lacked the histocompatability antigen HLA B27 one had an elevated level of $80 \mathrm{pg} / \mathrm{ml}(\mathrm{ng} / \mathrm{l})$, one had $0.5 \mathrm{pg} / \mathrm{ml}(\mathrm{ng} / \mathrm{l})$, and the third gave an anomalous result. Nine patients were receiving phenylbutazone: 3 of these had elevated levels, 5 were normal, and one gave an anomalous result.

\section{Discussion}

The circulating levels of biologically active parathyroid hormone (PTH) in normal subjects $(0 \cdot 2-12 \cdot 0 \mathrm{pg} / \mathrm{ml}=\mathrm{ng} / \mathrm{l})$ measured in this study accord with the levels calculated by Parsons et al. ${ }^{7}$ and found by others who have used this bioassay. ${ }^{9}{ }^{10}$ They are considerably less than those found by immunoassay, supporting the view that the latter measures biologically inactive fragments or modifications of the hormone.

Apart from 2 patients with mildly elevated PTH levels patients with osteoarthrosis had low normal circulating levels (Fig. 1). Thus the finding by McCarty et al. ${ }^{2}$ of elevated immunoreactive PTH in this condition may have been caused by the presence of biologically inactive fragments. Similarly they found elevated immunoreactive PTH levels in 15 of their 22 patients with chondrocalcinosis, whereas only 6 of our 21 patients showed elevated levels of biologically active PTH. However, it was clear (Fig. 1) that the range of values found in this group was very wide This range might be a reflection of wide fluctuations in individual patients during the course of the disease, as shown by the results on one patient whose level was high $(60 \mathrm{pg} / \mathrm{ml}=\mathrm{ng} / \mathrm{l})$ during an acute episode, but low $(2 \cdot 3 \mathrm{pg} / \mathrm{ml}=\mathrm{ng} / \mathrm{l})$ during a quiescent phase. Similar fluctuations in immunoreactive PTH levels were found by Phelps and Hawker. $^{5}$ Perhaps bioassay is capable of detecting fluctuations which lead to subtle changes in calcium homoeostasis sufficient perhaps to allow cartilage calcification to occur but which are not associated with measurable changes in serum calcium; or perhaps elevated levels occur only at intervals in each patient and such transient rises in PTH are related to excessive local concentrations of calcium that permit crystallisation. However this may be, it is of interest that Rynes and Merzig ${ }^{13}$ found that $67 \%$ of patients over the age of 60 who had surgically proved parathyroid adenomas had radiological evidence of chondrocalcinosis, as against $8 \%$ in a group of ageand sex-matched controls.

The finding of raised levels in 3 out of 6 patients with haemochromatosis was surprising. Only 2 of these 3 patients had chondrocalcinosis, though all 3 had insulin-dependent diabetes. Thus the diabetes may possibly be more relevant to their raised PTH levels than their chondrocalcinosis. However, Heath et al. ${ }^{14}$ in contrast to the results on experimental diabetes in the rat ${ }^{15}$ showed no increase in immunoreactive PTH in human insulin-dependent diabetes, even when poorly controlled.

The possibility that raised levels of bioactive PTH could be nonspecific and secondary to inflammatory joint disease does not appear to be supported by the 
finding of normal levels in the patients with rheumatoid arthritis, all of whom had clinical evidence of inflammation. While the number of patients is small, these normal findings are of interest in view of recent reports of both hypo- and hypercalcaemia in some rheumatoid patients. ${ }^{16} 17$

In the spondylitic patients a wide range of levels was recorded. With one possible exception all 5 females had normal circulating levels. Otherwise no correlation could be found with age, duration of the disease, or the presence of the histocompatability antigen HLA B27. The disease activity, assessed clinically as in the study of Fairney et al., ${ }^{6}$ did not appear to be correlated with PTH levels, nor did therapy with phenylbutazone, which is a known goitrogen. Our results (Fig. 1) indicated that, in contrast to the levels of immunoreactive PTH in this condition reported by Fairney et al., ${ }^{6} 17$ of our patients had normal levels and only about a third had markedly elevated circulating levels of bioactive hormone. However, our population seemed to be different from that studied by Fairney et al. ${ }^{6}$ in that the serum alkaline phosphatase was not elevated $(8 \cdot 32 \pm 2 \cdot 12$ $\mathrm{KA}$ units) in contrast to the findings in their group.

Although there were differences between our results and those of Fairney et al., ${ }^{6}$ we too found elevated or grossly elevated levels of PTH in 10 of our patients. In such patients the lack of overt hyperparathyroidism even in the presence of elevated levels of bioactive PTH might be accounted for by end-organ insensitivity, as suggested by Fairney et al. ${ }^{6}$ However, this does not seem to be the explanation either for the 4 samples (Fig. 3) which gave anomalously nonparallel responses at low dilution (but gave parallel responses at high dilution) or for the 3 that gave anomalously low, nonparallel responses. Thus the results with the plasma from these 7 patients were indicative of the presence in the plasma of some inhibitory influence that acted either on the hormone or on the receptor. This merits further, more detailed investigation.

Thus this study has shown a wide disparity between the circulating levels of biologically active parathyroid hormone and the levels of immunoreactive hormone previously reported by others. It has also drawn attention to the significant variations of the bioreactive hormone in the different conditions. The levels in most of the patients with osteoarthrosis were in the low range of normality, suggesting an even greater immuno- to biodissociation in this condition. The wide range of values found in patients with chondrocalcinosis might indicate that the hormonal concentration fluctuates greatly in this disease; this merits further study of the pathogenic role of the hormone. But the most surprising findings were the very diverse values found in ankylosing spondylitis, with the suspicion that in a proportion of these patients some interference with the biological effects of parathyroid hormone may exist.

We are grateful to the Arthritis and Rheumatism Council for Research for their support for this work. We are also grateful to $\mathrm{Dr}$ E. B. D. Hamilton and the Liver Unit at King's College Hospital for permission to study the patients with haemochromatosis under their care. We also thank the National Institute for Biological Standards and Control for supplying the standard preparation of parathyroid hormone.

\section{References}

${ }^{1}$ Bywaters E G L, Dixon A St J, Scott J T. Joint lesions of hyperparathyroidism. Ann Rheum Dis 1963; 22: 171-87.

2 McCarty D J, Silcox D C, Coe F, et al. Diseases associated with calcium pyrophosphate dihydrate crystal deposition. Am J Med 1974; 56: 704-14.

3 Hamilton E B D. Other metabolic arthropathies. In: Scott J T, ed. Copeman's Textbook of the Rheumatic Diseases. London and Edinburgh: Churchill Livingstone, 1978: section VII; 692.

4 Pritchard M H, Jessop J D. Chondrocalcinosis in primary hyperparathyroidism. Ann Rheum Dis 1977; 36: 146-51.

5 Phelps P, Hawker C D. Serum parathyroid hormone levels in patients with calcium pyrophosphate crystal deposition diseases. Arthritis Rheum 1973; 16: 590-6.

- Fairney A, Wotjulewski H, Hart F D. Parathyroid hormone measurement in ankylosing spondylitis. In: Talmage $R \mathrm{~V}$, Owen M, Parson J A, eds. Calcium Regulating Hormones. Amsterdam: Excerpta Medica, 1975: 72.

7 Parsons J A, Rafferty B, Gray D, et al. Pharmacology of parathyroid hormone and some of its fragments and analogues. In: Talmage R V, Owen M, Parsons J A, eds. Calcium Regulating Hormones. Amsterdam: Excerpta Medica, 1975: 33-9.

s Chambers D J, Dunham J, Zanelli J M, Parsons J A, Bitensky L, Chayen J. A sensitive bioassay of parathyroid hormone in plasma. Clin Endocr 1978; 9: 375-9.

- Fenton S, Somers S, Heath D A. Preliminary studies with the sensitive cytochemical assay for parathyroid hormone. Clin Endocrinol (Oxf) 1978; 9: 381-4.

10 Goltzman D, Henderson B, Loveridge N. Cytochemical bioassay of parathyroid hormone; characteristics of the assay and analysis of circulating forms. J Clin Invest 1980; 65: 1309-17.

${ }^{11}$ Bennett P H, Wood P H N. Population studies of the rheumatic diseases. Proceedings of the 3rd International Symposium, New York, 1966. Amsterdam: Excerpta Medica, 1968: 456.

12 Ropes M W, Bennett G A, Cobb S, Jacox R, Jessar R A. Diagnostic criteria for rheumatoid arthritis. 1958 revision. Ann Rheum Dis 1959; 18: 49-53.

13 Rynes R I, Merzig E G. Calcium pyrophosphate crystal deposition disease and hyperparathyroidism. A controlled, prospective study. J Rheumatol 1978; 5: 460-8.

14 Heath H III, Lambert P W, Service J S, Arnaud S B. Calcium homeostasis in diabetes mellitus. J Clin Endocrinol Metab 1979; 49: 462-6.

15 Schedl H P, Heath H III, Wenger J. Serum calcitonin and parathyroid hormone in experimental diabetes. Endocrinology 1978; 103: 1368-73.

${ }^{16}$ Bramble M G, Blake D R, White T, Sly J, Kerr D N S. Ionised calcium in rheumatoid arthritis: effect of non-steroidal antiinflammatory drugs. $\mathrm{Br}$ Med $J$ 1980; 281: 840-1.

17 Kennedy A C, Allam B F, Rooney P J. et al. Hypercalcaemia in rheumatoid arthritis: investigation of its causes and implications. Ann Rheum Dis 1979; 38: 401-12. 